\title{
Research on Membrane Structure Performance
}

\author{
Xuyu Tian ${ }^{1, *}$, Shuntao $\mathrm{Wu}^{1}$, Peiling $\mathrm{He}^{1}$, Xiong Zhou ${ }^{1}$, Zhaonian Bian ${ }^{1}$, Jinlin $\mathrm{Hu}^{1}$ \\ ${ }^{1}$ School of Architectural Engineering, Nanjing Institute of Technology, Nanjing, 211167, China
}

\begin{abstract}
Membrane materials and open-close roof buildings are new building materials and structural forms, which have developed rapidly in recent years. This new form of close combination of architecture and nature has enriched the connotation of architecture, exhibited the city, but also to the architectural design and construction has brought great challenges. Through the research and analysis of the performance of the membrane material, this study provides a reference for the design and construction of the domestic membrane material for the opening and closing roof structure.
\end{abstract}

\section{Introduction}

In the the Fifties or sixties of the 20th century, along with the revolution in material technology, people's requirements for the use of building functions gradually raised, known as the "fifth generation Building Materials" [1] of high-strength flexible film (hereinafter referred to as "membrane") into people's vision, membrane structure began to be used in temporary buildings. In the the early 1970s, glass fiber fabric as the substrate of the "permanent membrane" by the United States DuPont mainly developed by several companies and design units, so that the membrane structure is officially used in permanent buildings. Over the time, with the full study of membrane materials in some western countries and Japan, people have a deep understanding of their mechanical properties (tensile and tear resistance), making the space large-span curved membrane structure become Synonymous with "lightweight, beautiful, modern" architecture, and it has become one of the mainstream forms of opening and closing roof structures. Designers can design and build according to their wishes open and close roof film structure building, not only to achieve the architectural function and urban cultural heritage of the coordination and unity, but also to express the "unity of Heaven and Man" this kind of architectural and natural deep-seated unique views, is not only the foundation of architectural art schools, but also the embodiment of material concretization of harmonious society.

\section{Types and properties of membrane materials}

The membrane material can be divided into two categories, such as fabric film and foil film, according to the different structural structure.

Fabric film (Fig. 1) is a composite of fabric base material (hereinafter referred to as "substrate") and coating materials (hereinafter referred to as "coating") composite which has high strength and flexibility. Common substrates are polyester fiber, fiberglass, polypropylene fiber, polyamide amide fiber, etc., the common coating [2] has PVC (polyvinyl chloride), PTFE (polytetrafluoroethylene, commonly known as "Teflon") and so on. The mechanical properties of fabric film material like strength are mainly provided by the base material, and coating materials has fire prevention, antiaging and other physical and chemical properties.

Foil film is made of fluorine plastic. In recent years, the most commonly used material is ETFE membrane.

The study of performance of the following materials is around the PVC coating covering polyester fiber film, PTFE coating covered fiberglass base membrane and ETFE, which are commonly used in current open roof building.
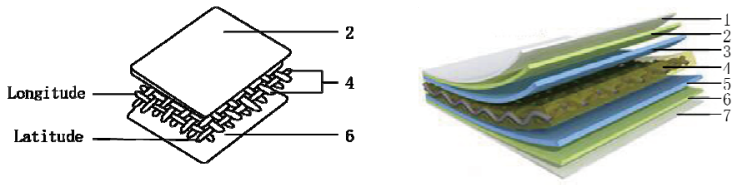

1- Surface coating,2- Main coating,3-Adhesive layer,4Fabricbase,5- Adhesive layer,6- Main coating,7- Surface coating

Fig. 1. Layered structure diagram of Fabric membrane

\section{PVC}

\subsection{Main properties of PVC}

The main properties of the four kinds of PVC membranes which surface treatment is different are compared in table 1 .

\subsection{Performance of PVC}


1) At room temperature, the stress-strain of PCV

divided into the elastic stage of the front line, the yield membrane under uniaxial tensile load (Fig. 2) is mainly

Table 1. Comparison of the main properties of four kinds of PVC membrane materials[3].

\begin{tabular}{|c|c|c|c|c|}
\hline Main performance & $\begin{array}{c}\text { Acrylic } \\
\text { surface PVC } \\
\text { film }\end{array}$ & $\begin{array}{l}\text { PVC film that can } \\
\text { splicing PVDF } \\
\text { surface }\end{array}$ & $\begin{array}{c}\text { PVC film that cannot } \\
\text { be spliced to PVDF } \\
\text { surface }\end{array}$ & $\begin{array}{l}\text { PVC film on PVF- } \\
\text { film surface }\end{array}$ \\
\hline Durable years & $3 \sim 7$ years & $8 \sim 12$ years & $10 \sim 15$ years & $10 \sim 15$ years \\
\hline Ageing resistance & low & Medium & high & High \\
\hline UV resistance & low & Medium & High & High \\
\hline Corrosion resistance & Low & Medium & High & High \\
\hline The degree of soft & high & High & high & High \\
\hline foldability & foldable & foldable & foldable & unfoldable \\
\hline $\begin{array}{l}\text { Performance of self- } \\
\text { cleaning }\end{array}$ & low & medium & high & Very high \\
\hline $\begin{array}{l}\text { Processing and } \\
\text { installing }\end{array}$ & easy & easy & hard & Very hard \\
\hline Splicing method & Joint directly & Joint directly & Polish/ & Polish/ \\
\hline Material cost & cheap & $\begin{array}{l}\text { Comparatively } \\
\text { expensive }\end{array}$ & expensive & expensive \\
\hline
\end{tabular}

stage and the second linear elastic stage.

The first line elastic stage is borne by the base and the coating, and the slope of the stress-strain curve depends on the yield ratio of the material. When the stress of the membrane material in the yield stage reaches the yield strength, the upward trend of the stressstrain curve is nonlinear, that is, the formation of the enhanced deformation stage, the coating begins to break and peel off, the intensity of the work gradually decreases; the elastic stage of the second line is when the coating breaks down to complete failure, and the upward trend of the stress-strain curve changes linearly, that is, the formation of the enhanced stress stage, the tension is all borne by the substrate, until the substrate begins brittle fracture failure, cannot meet the application of engineering materials.

Farrari1 1002T diretion
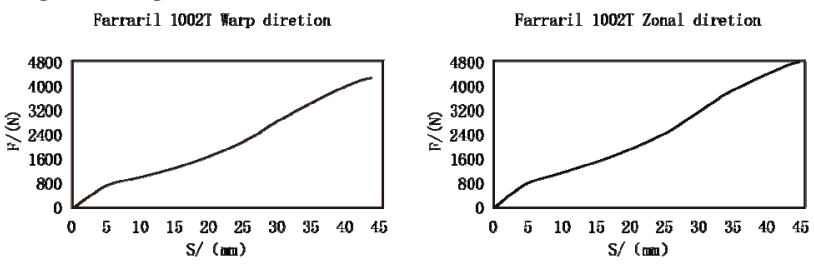

Fig. 2. Stress strain diagram of PVC.

2) Comparison of mechanical properties of commonly used PVC film materials (table 2).

\section{PTFE}

\subsection{Main properties of PTFE}

1) High and low temperature: Normal operation of the temperature is generally $180 \sim 260^{\circ} \mathrm{C}$.

2) Resist chemical corrosion:

Not only can withstand Wang Shui, concentrated hydrochloric acid, smoke sulfuric acid and other strong acidic substances and strong alkaline substances, but also strong oxidation agent, reducing agent and other organic solvents.

3) Excellent non-stickiness and smoothness:

Presented as infinity, the friction coefficient of thesurface is very small, the surface tension is only 0.019 $\mathrm{N} / \mathrm{m}$, most of the general material cannot adhere tothe surface. In the process of using them, they can be highly maintained the cleanliness of their own structure, not contaminated with dust and other pollutants.

4) Excellent weatherability:

The role of light performance is stable, not affected by ultraviolet and ozone and other optical emitting substances, in the relatively humid air and the surrounding environment, unaffected by microorganisms, can be exposed to the atmosphere for a long time, and the physical properties will not change. It has a long service life in the application of membrane materials.

5) Non-flammability:

The limit oxygen index is relatively high, belongs to the non-combustible material, the melting point is $275^{\circ} \mathrm{C}$.

6) Excellent dielectric properties and electrical insulation:

It will be penetrated within the voltage range of 25 40 Kv. Its dielectric nature does not change within the temperature range of the wide area, and the 
sensitivity to temperature is low, so it is often used in

high temperature resistant insulating materials.

Table 2. Stress strain diagram of PVC.

\begin{tabular}{|c|c|c|c|c|}
\hline Product performance index & VALMEX FR700 & VALMEX FR900 & FERRARI 1002T & FERRARI 702 \\
\hline \multirow{2}{*}{ Material } & Polyester & Polyester & Polyester & Polyester \\
\hline & Coated PVC & Coated PVC & Vinyl coated & Vinyl coated \\
\hline Warp tensile strength $(\mathrm{N} / 5 \mathrm{~cm})$ & 3000 & 4200 & 4200 & 2800 \\
\hline Latitude tensile strength $(\mathrm{N} / 5 \mathrm{~cm})$ & 3000 & 4200 & 4000 & 2800 \\
\hline Warp tear strength $/ \mathrm{N}$ & 300 & 500 & 550 & 300 \\
\hline Weft tear strength/N & 300 & 450 & 500 & 280 \\
\hline Bond strength $(\mathrm{N} / 5 \mathrm{~cm})$ & 100 & 125 & 120 & 100 \\
\hline
\end{tabular}

\subsection{Performance of PTFE}

1) The elongation deformation process of PTFE membrane under uniaxial tensile load (Fig. 3) is divided into three stages:

The first stage is the linear elastic deformation stage: The yield strength is $20 \mathrm{MPa}$ where the tensile stress of this stage material is between the $0 \sim 20 \%$ fracture strength, the elastic modulus is $900 \mathrm{MPa}$, the strain is about $1.7 \%$. The second stage is the nonlinear deformation stage, the yield strength is $25 \mathrm{MPa}$ where the tensile stress of this stage is between the $20 \% \sim 60 \%$ fracture strength, the strain is about $10.6 \%$, and the third stage is the linear enhanced stress stage where this stage of the membrane tensile stress exceeds $60 \%$ fracture strength, the coating completely loses the ability to withstand the tensile force, The tension is borne by the substrate directly, and this stage cannot meet the engineering requirements.

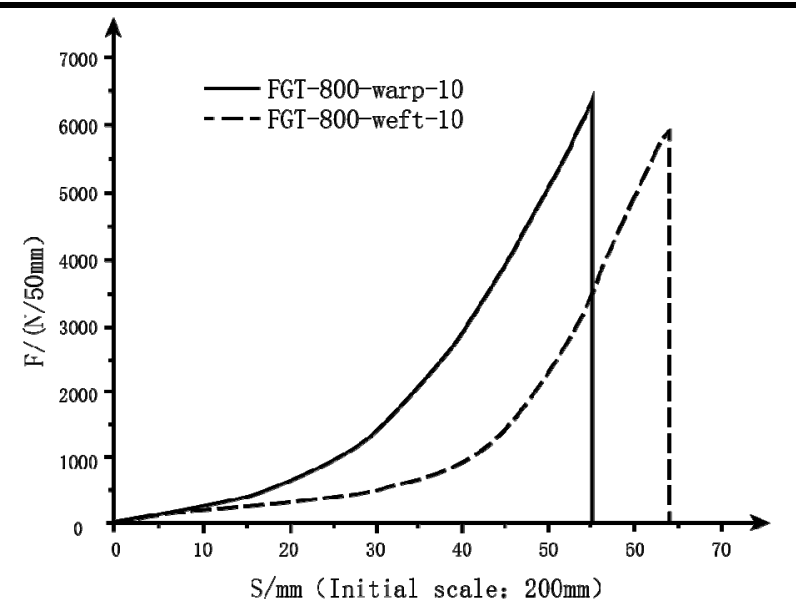

Fig. 3. Force displacement diagram of PTFE.

2) Comparison of mechanical properties of commonly used PTFE membrane materials (table 3)

Table 3. Comparison of mechanical properties of commonly used PTFE.

\begin{tabular}{ccccc}
\hline Product performance index & CHEMFAB & CHEMFAB & SKYTOP & VERSEIDAG \\
& SHEERFIIL I & SHEERFIIL II & FGT-800 & B18089 GF \\
\hline Material & glass fiber & glass fiber & glass fiber & glass fiber \\
Coated PTFE & Coated PTFE & Coated PTFE & 7800 & 7000 \\
Warp tensile strength & 7000 & 5500 & 6500 & 6000 \\
Latitude tensile strengt & 6100 & 4800 & 350 & 500 \\
Warp tear strength & 520 & 700 & 400 & 500 \\
Weft tear strength & 700 & 530 & & \\
\hline
\end{tabular}




\section{ETFE}

\subsection{Basic mechanical properties of ETFE}

1) Tensile strength:

Tensile strength greater than $40 \mathrm{MPa}$.

2) Break elongation:

Break elongation up to more than $300 \%$.

3) Stress-strain relationship $[4,5]$ :

At room temperature ETFE film is divided into complete elasticstage, yield stage, plastic strengthening stage (Fig. 4).Tensioning force is in the fully elastic stage below $20 \mathrm{MPa}$, the tensile film volume is up to $800 \sim 1000 \mathrm{Mpa}$, when the tensile stress near $25 \mathrm{MPa}$ will appear yield point, Tensioning force is in the yield stage at $20 \mathrm{Mpa} \sim 25 \mathrm{Mpa}$, and the tensile stress enters the plastic reinforcement stage after $25 \mathrm{MPa}$ until it breaks. Unlike other membrane materials' tensile curves (Fig. 5), the stress-strain curve of the ETFE membrane has the firstyield point and the second yield point, and its corresponding strength is the first yield strength and the second yield strength, respectively. The first type of long-term load of prestress and snow load which adopts the first yield strength, and the second type of short-term load, mainly wind load, adopts the second yield strength.

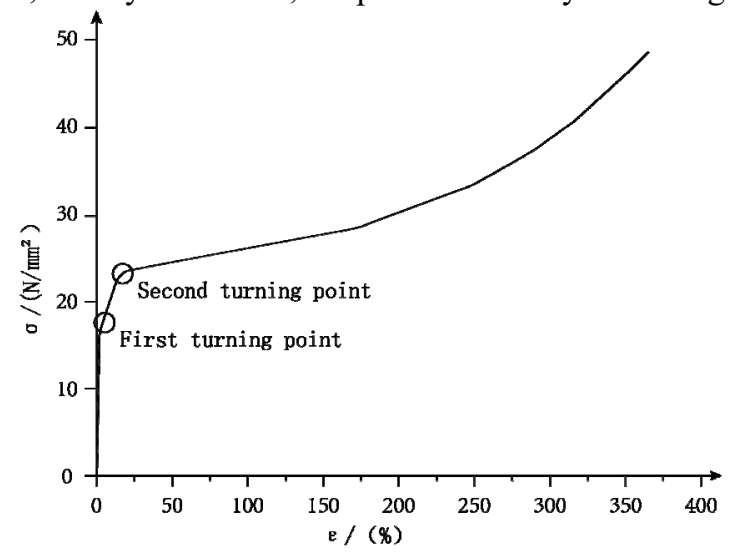

Fig. 4. Stress-Strain diagram of ETFE.

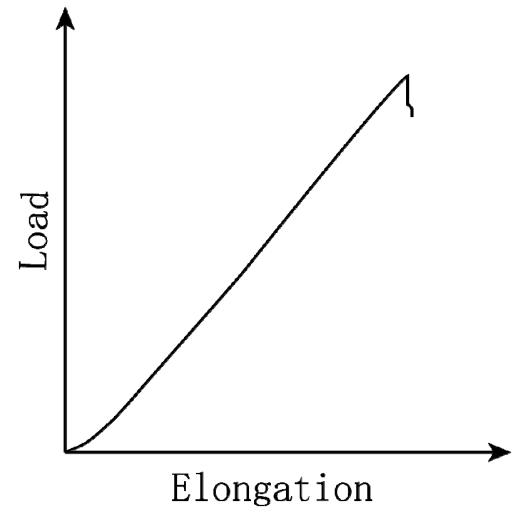

Fig. 5. Tensile curve of typical substrate.

\subsection{Other properties of ETFE}

The ETFE membrane is the most superior substitute material in the daylighting roof film material,which is compared with the properties of other membrane materials in table 4.

1) Thickness and quality:

Thickness is generally in the $0.05 \sim 0.25 \mathrm{~mm}$, with the increase in thickness, the film material becomes more brittle and hard and difficult to process; the density is about $1.75 \mathrm{~g} / \mathrm{cm}^{3}$.

2) Color and transmittance:

Usually colorless transparent or white. In practical engineering applications, according to the building effect, people can mix ETFE membrane with additives for dyeing or printing patterns, changing the transmittance of the membrane, blocking ultraviolet rays and other light transmission.

3) Self-cleaning performance:

With high anti-fouling, easy to cleancharacteristics, the surface can be descaled by the general natural rainwater scouring, manual surface cleaning generally four years once.

4) Fire Resistance:

It will melt in $200^{\circ} \mathrm{C}$, which fire rating reach $B 1$, DIN4102 standard, fire point is comparatively high, it

Table 4. Comparison of ETFE with other membrane materials[3].

\begin{tabular}{|c|c|c|c|c|c|c|}
\hline Performance & PFA & PEP & ETFE & CTFE & $\mathbf{P P} \cdot \mathbf{P E}$ & PVC \\
\hline Non-adhesive & excellent & excellent & excellent & normal & good & bad \\
\hline Antifouling & excellent & excellent & excellent & good & normal & bad \\
\hline Mildew proof & excellent & excellent & excellent & excellent & bad & bad \\
\hline Transparent & normal & excellent & excellent & excellent & normal & normal \\
\hline life & excellent & excellent & excellent & excellent & bad & bad \\
\hline Non-permeable steam & good & good & bad & excellent & bad & bad \\
\hline Airtight & excellent & excellent & excellent & bad & good & normal \\
\hline Drug resistance & excellent & excellent & good & good & normal & bad \\
\hline
\end{tabular}




\begin{tabular}{|c|c|c|c|c|c|c|}
\hline Heat resistance & excellent & excellent & excellent & good & bad & bad \\
\hline Flame retardant & excellent & excellent & excellent & excellent & bad & normal \\
\hline
\end{tabular}

will not occur spontaneous combustion generally and when it burn it will not drip.

5) Durability and weather resistance:

It has excellent anti-aging, stability, which can better adapt to a variety of environmental and climatic conditions and other factors. It has more than 25 years service life, operating temperature range between $200 \sim 150^{\circ} \mathrm{C}$, and melting point is about $275{ }^{\circ} \mathrm{C}$. In engineering practice, after more than 15 years explosion in harsh climatic conditions, the main performance of ETFE membrane has not changed. In the event of hail, even if the glass roof is smashed by hail, only a few tiny dents will be produced on the roof of the ETFE membrane.

\section{Conclusion}

Although the membrane structure building form is developing at a faster speed in China, the application prospect of the opening and closing roof structure is considerable. At present, the film produced in China is basically stronger than the film produced abroad, but the other properties (fire resistance, self-cleaning performance, service life) are far from each other. Therefore, China's membrane material research urgently needs to learn from foreign advanced technology, continuously improve the performance of membrane materials, improve the overall level of membrane structure research in China, and better combine membranes with domestic and open roof structure design.

\section{Acknowledgements}

The authors are grateful for the financial support from Nanjing Institute of Engineering 2018 Challenge Cup Cultivation Project (TZ20180018) and Provincial College Students Innovation and Entrepreneurship Training Program Project (201811276101H).

\section{References}

1. X. Chen, Z. G. Feng, J. New Building Materials, 7, 59-61(2004)

2. C. X. Xu, Y. Wu, J. Industrial Building, 6, 7983(2004)

3. W. Z. LONG, B. K. BAI, M. Chemical Industry Press, Beijing,2007)

4. L. Sun, T. T. Zhou, J. Li, J. Mechanics and Practice, 3, 89-90(2008)

5. C. X.Xu, C. X. Chen, R.J.Qian, Steel Construction, 6, 1-4(2003) 